\title{
A duração da pausa silente difere entre palavras de classe aberta ou fechada?
}

\section{Does the duration of silent pauses differ between words of open and closed class?}

\author{
Paula Renata Pedott', Letícia Bondezan Bacchin'1, Ana Manhani Cáceres-Assenço', Debora Maria Befi-Lopes ${ }^{1}$
}

\section{RESUMO}

Objetivo: Verificar se o tempo médio das pausas silentes difere para a classe das palavras (aberta ou fechada) e se há diferença entre esse tempo para crianças em desenvolvimento típico de linguagem e crianças com distúrbio específico de linguagem (DEL), em cada tipo de palavra. Métodos: Participaram da pesquisa 40 crianças em desenvolvimento típico de linguagem e 20 com DEL, com idade variando entre 7 e 10 anos. Cada sujeito elaborou 15 narrativas, baseadas em uma sequência de quatro cenas cada. Após a transcrição das amostras, as palavras foram classificadas como de classe aberta (substantivo, adjetivo, verbo, advérbio e numeral), ou fechada (artigo, preposição, pronome, conjunção e interjeição). Em um segundo momento, as amostras foram submetidas a um software para análise das pausas silentes, que permitiu o levantamento do tempo (milissegundos) das pausas imediatamente anteriores a cada uma dessas categorias gramaticais. Resultados: Em ambos os grupos, a pausa silente foi mais longa quando precedia as palavras de classe fechada, sendo que o grupo com DEL, em geral, apresentou pausas silentes mais longas que seus pares. Conclusão: A duração da pausa silente varia conforme a classe gramatical da palavra que será enunciada, sendo menor quando precede palavras de classe aberta. Além disso, o fato de os indivíduos com DEL apresentarem pausas silentes mais longas que seus pares, confirma a menor velocidade de seu processamento linguístico.

Descritores: Linguagem infantil; Transtornos do desenvolvimento da linguagem; Narração; Fonoaudiologia; Desenvolvimento da Linguagem

\begin{abstract}
Purpose: The study aim was to determine whether the mean duration of silent pauses depends on the word class (open or closed) and to compare the duration for each type of word between children with typical language development and children with specific language impairment (SLI). Methods: The study included 40 children with typical language development and 20 children with SLI, aged between 7 and 10 years. Each subject produced 15 story narratives based on a separate sequence of four pictures for each narrative. After the transcription of the samples, the words were classified as open class (noun, adjective, verb, adverb, and numeral) or closed class (article, preposition, pronoun, conjunction, and interjection). In the second phase of the study, the samples were analyzed using software specific to the analysis of silent pauses and the duration (milliseconds) of the pauses that occurred immediately before each of these grammatical categories was recorded. Results: In both groups, silent pauses were longer when preceding closed class words and individuals in the SLI group produced longer silent pauses than their peers did. Conclusion: The duration of a silent pause varied according to the grammatical class of the preceded word and it was shorter when followed by an open class word. In addition, the fact that individuals with SLI produce longer silent pauses than their peers confirms that their language processing is slower.
\end{abstract}

Keywords: Child language; Language development disorders; Narration; Speech, language and hearing sciences; Language development

Trabalho realizado no Laboratório de Investigação Fonoaudiológica em Desenvolvimento da Linguagem e suas Alterações, Departamento de Fisioterapia, Fonoaudiologia e Terapia Ocupacional, Faculdade de Medicina, Universidade de São Paulo - USP - São Paulo (SP), Brasil.

(1) Departamento de Fisioterapia, Fonoaudiologia e Terapia Ocupacional, Faculdade de Medicina, Universidade de São Paulo - USP - São Paulo (SP), Brasil. Conflito de interesses: Não

Contribuição dos autores: $P R P$ responsável pela tabulação e análise dos dados, levantamento da literatura e redação do manuscrito; $L B B$ responsável pela tabulação e análise dos dados, levantamento da literatura; $A M C A$ responsável pela revisão da tabulação e análise dos dados, levantamento da literatura, análise estatística, interpretação dos resultados e revisão do artigo. $D M B L$ responsável pela orientação, elaboração do projeto, aprovação da versão final do artigo.

Endereço para correspondência: Debora Maria Befi-Lopes. R. Cipotânea, 51, Cidade Universitária, São Paulo (SP), Brasil, CEP 05360-160.

E-mail: dmblopes@usp.br

Recebido em: 18/12/2013; Aceito em: 12/3/2014 


\section{INTRODUÇÃO}

A aquisição da linguagem é um processo complexo e gradual, que exige alta demanda cognitiva. Quando aspectos específicos da linguagem (especialmente os sintáticos e lexicais) ainda estão em desenvolvimento, há maior chance de ocorrência de rupturas durante a emissão da fala ${ }^{(1,2)}$.

Durante o desenvolvimento linguístico, as disfluências são aceitáveis, uma vez que podem refletir as incertezas da criança durante o planejamento da sentença. Sendo assim, propiciam um ganho de tempo para elaboração do enunciado e tendem a diminuir gradualmente, com a maturidade do sistema linguístico $^{(2)}$. Crianças com distúrbio específico de linguagem (DEL) não costumam apresentar essa diminuição, pois possuem dificuldades morfossintáticas que as levam a cometer mais disfluências, se comparadas à população em desenvolvimento típico de linguagem ${ }^{(3,4)}$.

Dentre as rupturas gagas, a pausa silente pode ser utilizada como estratégia de ganho de tempo para formulação do enunciado, sem adição de palavras ou sons ${ }^{(2)}$. Seu uso também é referido quando há uma sobrecarga de informações relacionadas ao processamento linguístico ${ }^{(5,6)}$.

Como a ocorrência de rupturas está associada aos elementos linguísticos, há estudos recentes que buscam compreender as relações entre as diferentes classes de palavras e os eventos disfluentes ${ }^{(7,8)}$. Devido às diferenças de aquisição e complexidade, estudos relataram maior número de rupturas em palavras de classe fechada (como artigos e preposições), que são adquiridas posteriormente e utilizadas como elementos de ligação entre palavras e frases sendo, portanto, mais abstratas ${ }^{(9-11)}$. Já as palavras de classe aberta (como substantivos e verbos), são adquiridas anteriormente e apresentam referenciais mais concretos, sendo mais facilmente relacionadas ao contexto e, portanto, há menos evidência de disfluência nessa classe de palavra ${ }^{(9,10)}$. Alguns autores utilizaram exames eletrofisiológicos para estudar o comportamento cerebral durante a utilização de palavras de classe aberta e fechada e concluíram que o seu processamento ocorre de forma distinta, em razão de suas diferentes funções e relações linguísticas ${ }^{(12,13)}$.

Dando continuidade à investigação da relação entre a classe gramatical e a pausa silente, este estudo teve por objetivo verificar se o tempo médio das pausas silentes difere para a classe das palavras (aberta ou fechada) e se há diferença entre esse tempo para crianças em desenvolvimento típico de linguagem e crianças com distúrbio específico de linguagem (DEL), em cada tipo de palavra.

\section{MÉTODOS}

O projeto foi aprovado pela Comissão de Análises de Projetos de Pesquisa (CAPPesq) do Hospital das Clínicas da Faculdade de Medicina, da Universidade de São Paulo (USP), sob parecer número 1150/09. Os responsáveis pelas crianças que atenderam aos critérios de inclusão neste estudo assinaram o Termo de Consentimento Livre e Esclarecido.

\section{Casuística}

O estudo contou com 60 sujeitos falantes do Português, com idade variando entre 7 e 10 anos. O pareamento dos grupos considerou a faixa etária e, para cada sujeito com DEL, havia dois sujeitos sem alteração de linguagem. O grupo com DEL (GDEL) foi composto por 20 crianças, sendo 14 do gênero masculino. O grupo em desenvolvimento típico de linguagem (GDTL) foi composto por 40 crianças, sendo 18 do gênero masculino.

Os critérios de inclusão do GDEL foram: apresentar desempenho em tarefas de inteligência não verbal dentro dos limites de normalidade; ter produção de fala inteligível; ter diagnóstico de distúrbio específico de linguagem, confirmado pelo desempenho abaixo do esperado em, pelo menos, dois dos seguintes testes padronizados de linguagem: vocabulário, fonologia e pragmática do $\mathrm{ABFW}^{(14)} \mathrm{e}$ extensão média do enunciado ${ }^{(15)}$.

A seleção dos sujeitos do GDTL foi realizada em uma escola estadual da zona oeste da cidade de São Paulo. Os critérios de inclusão foram: não apresentar processos fonológicos produtivos na avaliação da fonologia ${ }^{(16)}$; ter desempenho adequado em tarefas que envolvem consciência fonológica, leitura e escrita $^{(17)}$; apresentar rendimento acadêmico compatível com a faixa etária e série que frequenta, confirmado pela ficha escolar.

\section{Procedimentos}

Cada sujeito produziu 15 narrativas baseadas em histórias, representadas por uma sequência de quatro figuras ${ }^{(18)}$. Durante a coleta de dados, foi explicado aos participantes que a sequência de quatro figuras compunha uma história. A criança era solicitada a organizar as figuras e narrar a respectiva história, que era registrada em gravador digital. Para eliminar possíveis variáveis, como déficit de memória de curto prazo, as figuras ficaram visíveis para a criança durante todo o tempo de sua narração.

$\mathrm{O}$ arquivo com a gravação de cada história foi analisado acusticamente no software Audacity (1.3 Beta), que permitiu a marcação da duração de cada palavra enunciada pela criança e das pausas entre elas (transcrição da amostra). Todas as palavras foram classificadas como de classe aberta (substantivo, adjetivo, verbo, advérbio e numeral), ou fechada (artigo, preposição, pronome, conjunção e interjeição).

$\mathrm{O}$ arquivo foi, então, processado em um software, desenvolvido especificamente para calcular a duração das pausas silentes (em milissegundos), imediatamente anteriores a cada palavra. Esse software gerou um relatório para cada uma das 15 narrativas produzidas por cada sujeito, resultando no levantamento do tempo médio (em milissegundos) das pausas silentes anteriores a substantivos, adjetivos, verbos, advérbios, 
numerais, artigos, preposições, pronomes, conjunções e interjeições desta amostra. É importante ressaltar que, de acordo com os critérios adotados, consideramos como pausa silente todos os episódios de silêncio entre as palavras.

\section{Análise dos dados}

Foi realizada análise estatística descritiva e inferencial para responder aos objetivos deste estudo. O teste não paramétrico de Mann-Whitney foi utilizado para comparar o tempo médio das pausas silentes entre os grupos e o teste não paramétrico de Wilcoxon foi utilizado para a comparação entre as classes de palavras. Essa análise foi realizada no software SPSS versão 18 e o nível de significância adotado foi de 5\%.

\section{RESULTADOS}

A comparação entre as classes de palavras indicou que a pausa silente foi mais longa quando precedia as palavras de classe fechada, tanto para o GDTL ( $\mathrm{z}=-5,471, \mathrm{p}<0,001)$, quanto para o GDEL ( $\mathrm{z}=-3,435, \mathrm{p}=0,001)$ (Figura 1).

Dentre as palavras de classe aberta, as pausas silentes foram mais longas para o grupo com DEL, com exceção do numeral (Tabela 1).

Já dentre as palavras de classe fechada, o grupo com DEL teve pausas mais longas, com exceção da interjeição (Tabela 2).

\section{DISCUSSÃO}

Verificamos que a duração da pausa silente foi menor quando precedia palavras de classe aberta, em ambos os grupos. Por serem frequentes na fala materna e anteriormente adquiridas,

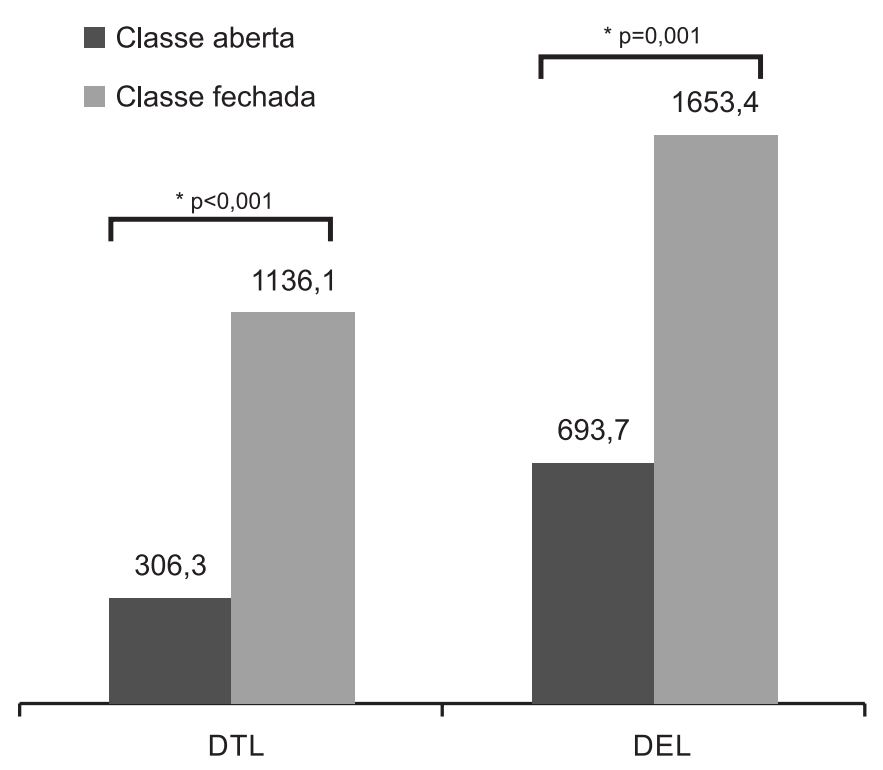

Figura 1. Tempo médio (em milissegundos) das pausas silentes anteriores a palavras de classe aberta e fechada em ambos os grupos

as palavras de classe aberta são utilizadas com mais constância e por mais tempo no cotidiano das crianças ${ }^{(2)}$. Esse contato contínuo e precoce propicia a solidificação do conceito, da semântica e da fonologia dessas palavras, o que leva ao acesso lexical mais rápido e preciso ${ }^{(19,20)}$.

Conforme amplia-se o vocabulário, a extensão do enunciado das crianças tende a aumentar concomitantemente e, desta forma, a introdução de palavras de classe fechada no enunciado torna-se necessária para que ocorra a conexão entre as palavras da sentença e entre as próprias sentenças. Sendo assim, por não possuírem referencial concreto e serem adquiridas posteriormente, é compreensível que o tempo médio das pausas silentes seja maior quando precede as palavras de classe fechada ${ }^{(21)}$.

Tabela 1. Comparação do tempo médio (em milissegundos) das pausas silentes anteriores a palavras de classe aberta

\begin{tabular}{|c|c|c|c|c|c|c|c|c|c|}
\hline \multirow{2}{*}{$\begin{array}{l}\text { Classe de } \\
\text { palavra }\end{array}$} & \multirow[b]{2}{*}{ Grupo } & \multirow[b]{2}{*}{ Média } & \multirow{2}{*}{$\begin{array}{l}\text { Desvio- } \\
\text { padrão }\end{array}$} & \multirow[b]{2}{*}{ Mediana } & \multicolumn{2}{|c|}{ Intervalo de confiança } & \multirow[b]{2}{*}{ U } & \multirow[b]{2}{*}{ Z } & \multirow[b]{2}{*}{ Valor de $p$} \\
\hline & & & & & $\begin{array}{c}\text { limite } \\
\text { inferior }\end{array}$ & $\begin{array}{c}\text { limite } \\
\text { superior }\end{array}$ & & & \\
\hline \multirow{2}{*}{ Substantivo } & DTL & 25,70 & 19,25 & 21,69 & 19,54 & 31,85 & \multirow{2}{*}{71,0} & \multirow{2}{*}{$-5,159$} & \multirow{2}{*}{$<0,001^{*}$} \\
\hline & DEL & 67,22 & 37,22 & 55,91 & 49,80 & 84,64 & & & \\
\hline \multirow{2}{*}{ Adjetivo } & DTL & 52,76 & 85,44 & 20,84 & 25,44 & 80,09 & \multirow{2}{*}{255,5} & \multirow{2}{*}{$-2,267$} & \multirow{2}{*}{$0,023^{*}$} \\
\hline & DEL & 190,74 & 301,31 & 112,61 & 49,72 & 331,76 & & & \\
\hline \multirow{2}{*}{ Verbo } & DTL & 89,05 & 45,91 & 76,06 & 74,36 & 103,73 & \multirow{2}{*}{144,0} & \multirow{2}{*}{$-4,014$} & \multirow{2}{*}{$<0,001^{*}$} \\
\hline & DEL & 185,71 & 94,90 & 172,96 & 141,30 & 230,12 & & & \\
\hline \multirow{2}{*}{ Advérbio } & DTL & 99,48 & 100,80 & 70,59 & 67,24 & 131,72 & \multirow{2}{*}{215,0} & \multirow{2}{*}{$-2,901$} & \multirow{2}{*}{$0,004^{*}$} \\
\hline & DEL & 182,52 & 142,70 & 136,79 & 115,73 & 249,30 & & & \\
\hline \multirow{2}{*}{ Numeral } & DTL & 39,33 & 134,43 & 0,00 & $-3,66$ & 82,32 & \multirow{2}{*}{328,0} & \multirow{2}{*}{$-1,393$} & \multirow{2}{*}{0,164} \\
\hline & DEL & 67,55 & 133,77 & 0,00 & 4,95 & 130,16 & & & \\
\hline \multirow{2}{*}{ Aberta geral } & DTL & 306,32 & 210,38 & 230,52 & 239,03 & 373,60 & \multirow{2}{*}{146,0} & \multirow{2}{*}{$-3,983$} & \multirow{2}{*}{$<0,001^{*}$} \\
\hline & DEL & 693,73 & 464,34 & 586,83 & 476,41 & 911,05 & & & \\
\hline
\end{tabular}

*Valores significativos $(p \leq 0,05)$ - Teste de Mann-Whitney

Legenda: $D T L=$ crianças em desenvolvimento típico de linguagem $(n=40) ; D E L$ = crianças com distúrbio específico de linguagem $(n=20), U=$ estatística do teste de Mann-Whitney; $Z$ = escore $Z$ 
Tabela 2. Comparação do tempo médio (em milissegundos) das pausas silentes anteriores a palavras de classe fechada

\begin{tabular}{|c|c|c|c|c|c|c|c|c|c|}
\hline \multirow{2}{*}{$\begin{array}{l}\text { Classe de } \\
\text { palavra }\end{array}$} & \multirow[b]{2}{*}{ Grupo } & \multirow[b]{2}{*}{ Média } & \multirow[b]{2}{*}{$\begin{array}{l}\text { Desvio- } \\
\text { padrão }\end{array}$} & \multirow[b]{2}{*}{ Mediana } & \multicolumn{2}{|c|}{ Intervalo de confiança } & \multirow[b]{2}{*}{ U } & \multirow[b]{2}{*}{ Z } & \multirow[b]{2}{*}{ Valor de $p$} \\
\hline & & & & & $\begin{array}{c}\text { limite } \\
\text { inferior }\end{array}$ & $\begin{array}{c}\text { limite } \\
\text { superior }\end{array}$ & & & \\
\hline \multirow{2}{*}{ Artigo } & DTL & 97,86 & 70,85 & 76,52 & 75,20 & 120,52 & \multirow{2}{*}{81,0} & \multirow{2}{*}{$-5,002$} & \multirow{2}{*}{$<0,001^{*}$} \\
\hline & DEL & 244,79 & 118,99 & 212,56 & 189,10 & 300,48 & & & \\
\hline \multirow{2}{*}{ Preposição } & DTL & 75,76 & 72,26 & 56,96 & 52,65 & 98,87 & \multirow{2}{*}{124,0} & \multirow{2}{*}{$-4,328$} & \multirow{2}{*}{$<0,001^{*}$} \\
\hline & DEL & 169,40 & 100,38 & 136,74 & 122,42 & 216,38 & & & \\
\hline \multirow{2}{*}{ Pronome } & DTL & 179,49 & 245,50 & 94,88 & 100,98 & 258,01 & \multirow{2}{*}{271,0} & \multirow{2}{*}{$-2,023$} & \multirow{2}{*}{$0,043^{*}$} \\
\hline & DEL & 283,29 & 271,01 & 214,76 & 156,46 & 410,13 & & & \\
\hline \multirow{2}{*}{ Conjunção } & DTL & 309,45 & 213,52 & 261,41 & 241,16 & 377,73 & \multirow{2}{*}{232,0} & \multirow{2}{*}{$-2,634$} & \multirow{2}{*}{$0,008^{*}$} \\
\hline & DEL & 478,09 & 269,55 & 425,85 & 351,94 & 604,24 & & & \\
\hline \multirow{2}{*}{ Interjeição } & DTL & 473,49 & 578,62 & 351,68 & 288,44 & 658,54 & \multirow{2}{*}{369,5} & \multirow{2}{*}{,- 479} & \multirow{2}{*}{0,632} \\
\hline & DEL & 477,80 & 379,28 & 366,30 & 300,29 & 655,31 & & & \\
\hline \multirow{2}{*}{$\begin{array}{l}\text { Fechada } \\
\text { geral }\end{array}$} & DTL & 1136,05 & 797,03 & 928,95 & 881,15 & 1390,96 & \multirow{2}{*}{221,0} & \multirow{2}{*}{$-2,807$} & \multirow{2}{*}{$0,005^{*}$} \\
\hline & DEL & 1653,37 & 808,90 & 1435,62 & 1274,80 & 2031,94 & & & \\
\hline
\end{tabular}

*Valores significativos ( $\mathrm{p} \leq 0,05)$ - Teste de Mann-Whitney

Legenda: $D T L=$ crianças em desenvolvimento típico de linguagem $(n=40) ; D E L=$ crianças com distúrbio específico de linguagem ( $n=20)$, $U=$ estatística do teste de Mann-Whitney; $Z$ = escore $Z$

Quanto à duração dessas pausas, que também foi maior para crianças com DEL nas duas classes de palavras, ressalta-se que, como essas crianças usualmente apresentam prejuízo na memória e aquisição lexical mais lenta, que pode estar relacionada tanto a uma dificuldade simbólica (prejudicando a categorização dos traços semânticos desses vocábulos), quanto a uma desorganização do sistema fonológico (dificultando a representação fonológica das mesmas), o acesso lexical pode ser mais impreciso. Porém, apesar desse padrão, as palavras de classe aberta continuam a compor a categoria gramatical mais facilmente adquirida e utilizada por esses sujeitos, devido a sua grande carga de representação concreta ${ }^{(15,22)}$.

Com relação às palavras de classe fechada, é sabido que essa população, que já apresenta déficits em outras etapas do processamento da linguagem, tem mais dificuldade para compreender a necessidade e o contexto correto da sua utilização. Assim, apresentam falhas na formação das regras gramaticais da língua e produzem sentenças curtas e sem refinamento sintático, necessitando de maior tempo para processar essas palavras conectoras, o que evidencia a razão pela qual as pausas que as precedem sejam maiores ${ }^{(21)}$.

O fato de a população com DEL ter evidenciado o uso de pausas silentes mais longas do que as crianças em desenvolvimento típico, em todas as classes gramaticais estudadas, com exceção do numeral e da interjeição, reflete a dificuldade de aquisição lexical dessa população e reflete também, claramente, as incertezas desses sujeitos quanto à formulação linguística em atividade discursiva ${ }^{(2,21)}$. Possivelmente, a ausência de diferença estatística em relação ao numeral e à interjeição ocorreu devido ao fato do estímulo eliciador das narrativas não ter favorecido a produção desse tipo de palavra em nenhum dos grupos, o que interferiu na análise dos dados relativos a essas classes de palavras.

A partir desses dados, verificou-se intrínseca relação entre a aquisição e utilização das classes gramaticais e seu impacto na fluência da fala em crianças em idade escolar, tendo sido possível verificar a diferença entre o nível de domínio linguístico em cada uma das populações estudadas.

Os resultados contribuem para a avaliação diagnóstica, ao indicarem que um discurso com pausas longas entre as palavras seja indicativo de comprometimento nas habilidades de elaboração discursiva. Portanto, o processo de intervenção terapêutica de crianças com alteração do desenvolvimento da linguagem deve considerar a necessidade de aprimorar o discurso desses sujeitos, permitindo que sejam capazes de transmitir sua mensagem com maior clareza e de uma forma mais elaborada.

\section{CONCLUSÃO}

A duração da pausa silente varia conforme a classe gramatical da palavra que será enunciada, sendo menor quando precede palavras de classe aberta. Além disso, o fato de os indivíduos com DEL apresentarem pausas silentes mais longas que seus pares, confirma a menor velocidade de seu processamento linguístico.

\section{AGRADECIMENTOS}

À Fundação de Amparo à Pesquisa do Estado de São Paulo (FAPESP), pelo apoio concedido para realização dessa pesquisa, sob processos números 2009/14045-0, 2010/07613-0 e 2010/07637-6. 


\section{REFERÊNCIAS}

1. Rispoli M. Changes in the nature of sentence production during the period of grammatical development. J Speech Lang Hear Res. 2003;46(4):818-30. http://dx.doi.org/10.1044/1092-4388(2003/064)

2. Guo LY, Tomblin JB, Samelson V. Speech disruptions in the narratives of English-speaking children with specific language impairment. J Speech Lang Hear Res. 2008;51(3):722-38. http://dx.doi. org/10.1044/1092-4388(2008/051)

3. Rodrigues A, Befi-Lopes DM. Memória operacional fonológica e suas relações com o desenvolvimento da linguagem infantil. Pró-fono Rev Atual Cient. 2009;21(1):63-8. http://dx.doi.org/10.1590/S010456872009000100011

4. Befi-Lopes DM, Bacchin LB, Pedott PR, Cáceres-Assenço AM. Complexidade da história e pausas silentes em crianças com e sem distúrbio específico de linguagem. CoDAS. 2013;25(4):325-9. http:// dx.doi.org/10.1590/S2317-17822013000400005

5. Krivokapi J. Prosodic planning: effects of phrasal length and complexity on pause duration. J Phon. 2007;35(2):162-79. http://dx.doi. org/10.1016/j.wocn.2006.04.001

6. MacGregor LJ, Corley M, Donaldson DI. Listening to the sound of silence: disfluent silent pauses in speech have consequences for listeners. Neuropsychologia. 2010;48(14):3982-92. http://dx.doi.org/10.1016/j. neuropsychologia.2010.09.024

7. Au-Yeung J, Gomez IV, Howell P. Exchange of disfluency with age from function words to content words in Spanish speakers who stutter. J Speech Lang Hear Res. 2003;46(3):754-65. http://dx.doi. org/10.1044/1092-4388(2003/060)

8. Juste FS, Sassi FC, Andrade CR. Exchange of disfluency with age from function to content words in Brazilian Portuguese speakers who do and do not stutter. Clin Linguist Phon. 2012;26(11-12):946-61. http:// dx.doi.org/10.3109/02699206.2012.728278

9. Juste FS, Andrade CRF. Speech disfluency types of fluent and stuttering individuals: age effects. Folia Phoniatr Logop. 2011;63(2):5764. http://dx.doi.org/10.1159/000319913

10. Savage C, Howell P. Lexical priming of function words and content words with children who do, and do not, stutter. J Commun Disord. 2008;41(6-5):459-84. http://dx.doi.org/10.1016/j.jcomdis.2008.01.004

11. Befi-Lopes DM, Pedott PR, Bacchin LB, Cáceres AM. Relação entre pausas silentes e classe gramatical em narrativas de crianças com distúrbio específico de linguagem. CoDAS. 2013;25(1):64-9. http:// dx.doi.org/10.1590/S2317-17822013000100012
12. Liu B, Jin Z, Wang Z, Wu G. Chinese function words grammaticalized from content words: evidence from ERPs. J Neurolinguist. 2010;23(6):663-75. http://dx.doi.org/10.1016/j. jneuroling.2010.07.002

13. Maxfield ND, Lyon JM, Silliman ER. Disfluencies along the garden path: brain electrophysiological evidence of disrupted sentence processing. Brain Lang. 2009;111(2):86-100. http://dx.doi.org/10.1016/j. band1.2009.08.003

14. Andrade CRF, Befi-Lopes DM, Fernandes FDM, Wertzner HF. ABFW: teste de linguagem infantil nas áreas de fonologia, vocabulário, fluência e pragmática. 2a ed. Barueri: Pró-Fono; 2004.

15. Araujo K. Desempenho gramatical de criança em desenvolvimento normal e com Distúrbio Específico de Linguagem [tese de doutorado]. São Paulo: Universidade de São Paulo; 2007.

16. Wertzner HF. Fonologia. In: Andrade CRF, Befi-Lopes DM, Fernandes FDM, Wertzner HF. ABFW: teste de linguagem infantil nas áreas de fonologia, vocabulário, fluência e pragmática. 2a ed. Barueri: Pró-Fono; 2004. Capítulo 1, p. 5-32.

17. Andrade C, Befi-Lopes D, Fernandes F, Wertzner H. Manual de avaliação de linguagem do serviço de fonoaudiologia do Centro de Saúde Escola Samuel B. Pessoa. São Paulo: Centro de Saúde Escola Samuel B. Pessoa; 1997. p. 127.

18. Perissinoto J, editor. Conhecimentos essenciais para atender bem a criança com autismo. São José dos Campos: Pulso; 2003. Capítulo 5, Avaliação fonoaudiológica da criança com autismo; p. 45-55.

19. Richtsmeier PT, Gerken L, Goffman L, Hogan T. Statistical frequency in perception affects children's lexical production. Cognition. 2009;111(3):372-7. http://dx.doi.org/10.1016/j.cognition.2009.02.009

20. Clark E. Adult offer, word-class, and child uptake in early lexical acquisition. First Lang. 2010;30(3-4):250-69. http://dx.doi. org/10.1177/0142723710370537

21. Castilho A. Fundamentos teóricos da gramática do português culto falado no Brasil: sobre o segundo volume, classes de palavras e as construções gramaticais. Alfa. 2007;51(1);99-135.

22. Gândara JP, Befi-Lopes DM. Tendências da aquisição lexical em crianças em desenvolvimento normal e crianças com alterações específicas no desenvolvimento da linguagem. Rev Soc Bras Fonoaudiol. 2010;15(2):297-304. http://dx.doi.org/10.1590/S151680342010000200024 